\title{
Integrando esfuerzos para la investigación científica universitaria
}

\author{
Lelany Pineda García
}

Una de las funciones fundamentales de las universidades es propiciar la creación de nuevos conocimientos a través de la investigación científica, tecnológica, humanística y social. Esta función se ha convertido en el centro de la evaluación de las universidades en muchos países del mundo.

La Universidad Nacional Autónoma de Honduras (UNAH) ha promovido intensamente, a partir de la IV reforma, los espacios para generar nuevas formas de investigación que aún no habían sido explotadas del todo ya que es menester de las reformas educativas incentivar, difundir y extender la investigación y sus tipos, formas y ámbitos que no han sido tratados. Por ello, la investigación necesita de nuevas formas de apropiación de la realidad, de observar y dimensionar el entorno, pues este es el punto de partida de la investigación; problematizar para poder crear y transformar.

En este sentido, las escuelas de salud tienen nuevas oportunidades para emprender transformaciones en su concepción educativa, en los currículos y la pedagogía con respecto a los escenarios de formación clínica en la provisión de servicios de salud, en proyectos comunitarios y en investigación.

La Facultad de Ciencias Médicas de la UNAH, comprometida con la promoción del conocimiento científico universal y la gestión de políticas públicas en salud, ha identificado la necesidad de crear una unidad académica que se focalice en temas y problemas del área de la salud, a través de la experiencia académica superior que por afinidad le corresponde. Es así que se propone la creación del Instituto de Investigación en Ciencias de la Salud (IICS) como un centro académico encargado de la investigación científica, desarrollo e innovación científica-tecnológica vinculada a la docencia especializada.

El propósito de la creación del IICS es impulsar la investigación de excelencia en Ciencias de la Salud y Biomedicina, con una atención particular de la investigación traslacional, término novedoso que tiene como propósito acelerar el paso de los hallazgos científicos realizados por la investigación básica hacia su aplicación clínica. En otras palabras, significa trasladar los conocimientos logrados en el

\footnotetext{
${ }^{1}$ Universidad Nacional Autónoma de Honduras. Facultad de Ciencias Médicas, Coordinadora del Instituto de Investigación en Ciencias de la Salud (IICS). Correo electrónico: lelanypineda@unah.edu.hn;

lelanypineda@yahoo.com
} 
laboratorio hacia una aproximación terapéutica o de prevención de enfermedades, favoreciendo el conocimiento aplicado y colaborando en el desarrollo de enseñanzas de nuevas metodologías en elárea de pre y posgrado.

Los procesos experimentados en los últimos años, la tecnología y los conocimientos que se han venido produciendo en el ámbito de la investigación biomédica, están permitiendo sustituir el viejo concepto de enfermedad, por el más moderno y estimulante que es de salud preventiva. El IICS pretende, entonces, ser exponente de estos cambios y consolidar las propuestas científicas de la biomedicina afrontando el reto de avanzar en la calidad de vida de los ciudadanos, a través de una iniciativa institucional encaminada a convertir a las ciencias de la salud en referente internacional de la docencia e investigación sanitaria.

En el IICS se estimulará la investigación y la formación de recursos humanos en aspectos básicos y preclínicos. Asimismo, se llevarán a cabo programas de trabajo a partir de proyectos de investigación con financiación externa e interna, conectando la investigación fundamental con la investigación aplicada. Además, se priorizará la producción de tesis de licenciatura, maestría y de doctorado en las distintas líneas de investigación.

Un elemento importante de la misión del IICS es vincular acciones con la comunidad en donde se encuentra inmerso. Por ello, se realizarán proyectos de investigación en asociación con el Hospital Escuela Universitario (HEU), en el marco del traspaso administrativo del Gobierno de la República a la UNAH, cuyas nuevas autoridades han renovado el viejo concepto de los hospitales como instituciones que proveen atención a pacientes con el fin de recuperar la salud, por un concepto de avanzada que se han apropiado en su propia misión, la cual es: "Ser una institución pública de servicios especializados de salud integral, oportuna y de alta calidad, siendo además una institución que contribuye al desarrollo científico de la salud y a la formación de recursos humanos a través de un proceso que incorpora las nuevas tendencias de atención en salud con una administración eficiente y transparente".

Este paso cualitativo institucional del HEU y los procesos de vinculación universidad-sociedad permiten brindar nuevos enfoques y puentes de asociación, en donde la actividad clínica y la academia avancen en un sistema de buenas relaciones funcionales. Mucho del trabajo de los profesores de Medicina e investigadores no puede darse sin una actividad clínica y los gerentes de salud reconocen que los estándares de atención no progresan sin la investigación académica y la experimentación de las escuelas médicas. 
Particularmente, el HEU ofrece un conjunto de escenarios y ambientes académicos que permiten una diversidad apreciable de actividades docentes, por ejemplo, clases presenciales, trabajo en grupos, campos de práctica clínica, rondas clínicas, manejo y seguimiento de pacientes, laboratorios y unidades de apoyo diagnóstico, administración hospitalaria; sumado a ello que se puedan proponer iniciativas que permitan identificar tensiones y compatibilidades entre las normas y guías nacionales e institucionales de atención clínica y los procesos formativos en el marco del modelo académico con el propósito de buscar el perfeccionamiento.

En este orden de ideas, tanto el IICS como el HEU formularán las políticas de investigación con lineamientos sobre las condiciones materiales para la investigación, el desarrollo y consolidación de los grupos de investigación en pregrado y posgrado, comunicación, publicaciones y divulgación, utilización del conocimiento, seguimiento y evaluación. Destaca también la promoción del HEU como un escenario privilegiado para avanzar en la investigación básica, clínica, de salud pública y ciencias sociales de forma integrada.

El IICS tiene dentro de su propuesta tres líneas prioritarias de investigación completamente compatibles con los procesos llevados a cabo en el HEU:

1. Enfermedades crónicas no transmisibles y estilos de vida

a. Líneas de investigación: enfermedades metabólicas y disfunción endotelial, neurociencia/neurología, oncología

2. Enfermedades transmisibles y promoción de la salud

a. Línea de investigación: enfermedades infecciosas

3. Salud mental y violencia

a. Línea de investigación: psiquiatría

b. Brecha de tratamiento de salud mental

Asimismo, se propone un eje transversal que incluye la innovación clínica en apoyo a la vigilancia de la salud. Además, se cuenta con las primeras infraestructuras de los laboratorios de investigación en el área del banco genético y laboratorio de histocompatibilidad. Por supuesto, estas líneas de investigación son susceptibles de ampliarse a otros escenarios de acuerdo a las propuestas de investigación. 
Como se ha planteado, el HEU se inscribe plenamente en las políticas de desarrollo académico de la UNAH en general y son de especial interés las líneas de desarrollo académico para el proceso de formación de los estudiantes. De estas líneas pueden destacarse los principios (excelencia académica, formación integral, formación investigativa, interdisciplinariedad y flexibilidad), las prácticas académicas especiales, las líneas de profundización y la articulación entre niveles de formación; destacándose los enfoques, grupos interdisciplinarios, la calidad y excelencia académica.

Es previsible que esta articulación académica-asistencial-investigación pueda obtener un impacto en términos de calidad y excelencia académica a través de:

1. La conformación de centros de excelencia.

2. El aporte a los ejes centrales de cada uno de los programas de pregrado y posgrado, en términos curriculares y pedagógicos, por medio de la determinación precisa de las competencias clínicas, profesionales e investigativas y el trabajo académico en equipos interdisciplinarios, en el manejo de los pacientes, los servicios de salud, la salud familiar y comunitaria.

3. La investigación y desarrollo pedagógico.

4. La investigación clínica, expandiendo sus dominios a una biomedicina que implique salud pública, la salud de las familias y las comunidades.

5. La investigación para el desarrollo tecnológico del sector salud y las respectivas políticas nacionales como referente.

6. Los procesos de traducción y mediación del conocimiento para incidir en las políticas de salud, con énfasis en el aseguramiento, tarifas de servicios, paquetes de servicios y prestadores de servicios, entre otros aspectos.

7. La confección de guías y protocolos de atención institucional, servicios de prevención y promoción de la salud.

El fin de todo este esfuerzo institucional es fortalecer y crear una masa crítica apropiada de investigadores biomédicos (básicos y clínicos) que puedan desarrollar el IICS y apoyar fuertemente al HEU, logrando la colaboración para impulsar la investigación en el país.

Como punto final, se establece que la tecnología por sí sola no contribuirá al avance de las grandes preguntas de la investigación biomédica, el éxito depende de realizar las preguntas con sentido biológico y médico y estas preguntas surgirán de la colaboración estrecha entre investigadores básicos y clínicos. 


\section{BIBLIOGRAFÍA}

Cabo Salvador, Javier. (2010) Investigación traslacional. Gestión sanitaria integral: pública y privada. Disponible en: $h$ ttp: //www. gestion-sanitaria.com Hesselbart Marquez, Carlos. (2009). Futuro de la medicina traslacional en cáncer. Cancerología, 47, 8.

OPS-OMS. (1992). La garantía de calidad. Acreditación de hospitales para América Latina y el Caribe. Desarrollo y fortalecimiento de los sistemas locales de salud. HSD/SILOS, 13.

Porter, Michael. (2006). Redefining Health Care: Creating Value-Based Competition on Results. Estados Unidos: Harvard Business School Publishing.

Sounding Board. (November 1997). The social missions of academic health centers. The New England Journal of Medicine, 337(21), 1550-1153.

Weiner, Bryan J.; Culbertson, Richard; Jones, Robert F. and Dickler, Robert. (February 2001). Organizational Models for Medical School-Clinical Enterprise Relationships. Academic Medicine, (76)2, 113-124. 